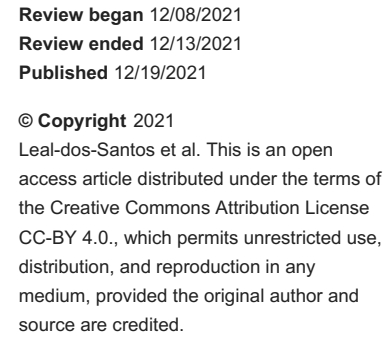

\section{A Thorn in a Haystack: A Rare Case of Septic Arthritis}

\author{
Marta Leal-dos-Santos ${ }^{1}$, José N. Ferreira ${ }^{2}$, David Peres ${ }^{3}$, Carlos Palos ${ }^{4}$ \\ 1. Infectious Diseases Department, Hospital de Curry Cabral-Centro Hospitalar Universitário de Lisboa Central, Lisbon, \\ PRT 2. Orthopaedics Department, Hospital Beatriz Ângelo, Loures, PRT 3. Infection and Antibiotic Resistance \\ Prevention and Control Unit, Hospital Pedro Hispano-Unidade Local de Saúde Matosinhos, Matosinhos, PRT 4. \\ Infection and Antibiotic Resistance Prevention and Control Unit, Hospital Beatriz Ângelo, Loures, PRT
}

Corresponding author: Marta Leal-dos-Santos, lealsantos.marta@gmail.com

\section{Abstract}

Pantoea agglomerans is a facultative anaerobe and environmental bacterium that could be a cause of opportunistic human infections, especially in wound infection with plant material. Arthritis or synovitis secondary to penetrating plant thorn injuries is not frequently reported. We present the case of a 35-yearold otherwise healthy male with a bramble thorn penetrating injury of the left knee. P. agglomerans was isolated from the synovial fluid. The patient was treated with amoxicillin/clavulanate according to sensitivity testing. This case highlights the importance of precise and thorough medical history, especially for less common presentations, as well as source control.

Categories: Infectious Disease, Orthopedics

Keywords: pantoea agglomerans, foreign body, gram negative, septic arthritis, thorn injurie

\section{Introduction}

Pantoea agglomerans is a facultative anaerobe and environmental bacterium of the family Enterobacterales found in plants, the earth, and water, and occasionally in wounds of animals [1,2]. P. agglomerans, though not an obligate infectious agent in humans, could be a cause of opportunistic human infections, especially in wound infection with plant material [3]. Arthritis or synovitis secondary to penetrating plant thorn injuries is not frequently reported. Historically, it is considered aseptic and treated with removal of the intra-articular foreign body and affected synovial lining [4]. To our knowledge, there have been only eight case reports in the literature (11 patients), which shows that this rare type of arthritis or synovitis can be difficult to diagnose because of an insidious onset after an apparently trivial injury.

\section{Case Presentation}

We present the case of a 35-year-old otherwise healthy male, including no immunosuppressant conditions or medications, with a bramble thorn penetrating injury of the left knee. The patient presented for two occasions to the emergency department, one week apart. Firstly, the patient complained of pain and swelling of the left knee, with limited flexion and inability to walk, and was started on amoxicillin/clavulanate and non-steroid anti-inflammatory drugs. On a second occasion, he presented with persistent pain, swelling, and reduced range of motion but was able to walk with aid and apparently with the presence of liquid and was admitted to the hospital. On physical examination and on both occasions, he was afebrile, but on the second visit to the emergency room, he showed a tense, swollen knee joint with redness and tenderness. A millimetric entry point was identified on the anterolateral surface of the knee, but no foreign body was visible. Septic arthritis was considered, and arthrocentesis was performed, revealing a turbid yellow fluid with 76,720 leukocytes/mL with $98 \%$ polymorphonuclears. Gram stain was negative. Blood tests showed an elevated C-reactive protein of $17.6 \mathrm{mg} / \mathrm{dL}$ (normal value $<0.5 \mathrm{mg} / \mathrm{dL}$ ), with leucocytosis $\left(11.88 \times 10^{9} / \mathrm{L}\right.$, normal range 4.00-10.00 $\times 10^{9} / \mathrm{L}$ ) and no neutrophilia. Computed tomography showed a significant amount of fluid with subquadricipital internal septa (Figures 1-3). 


\section{Cureus}

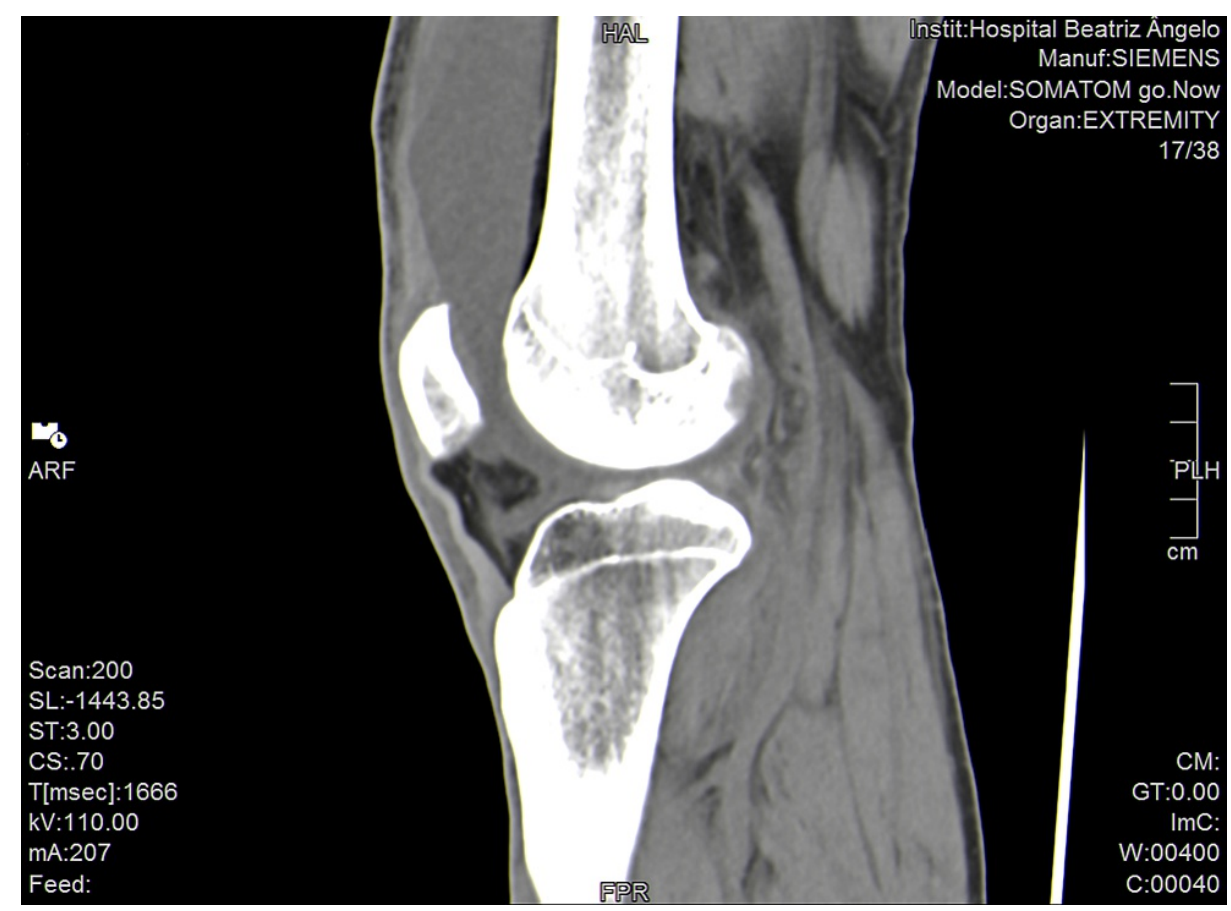

FIGURE 1: Increased articular liquid extending to subquadricipital space (sagital view).

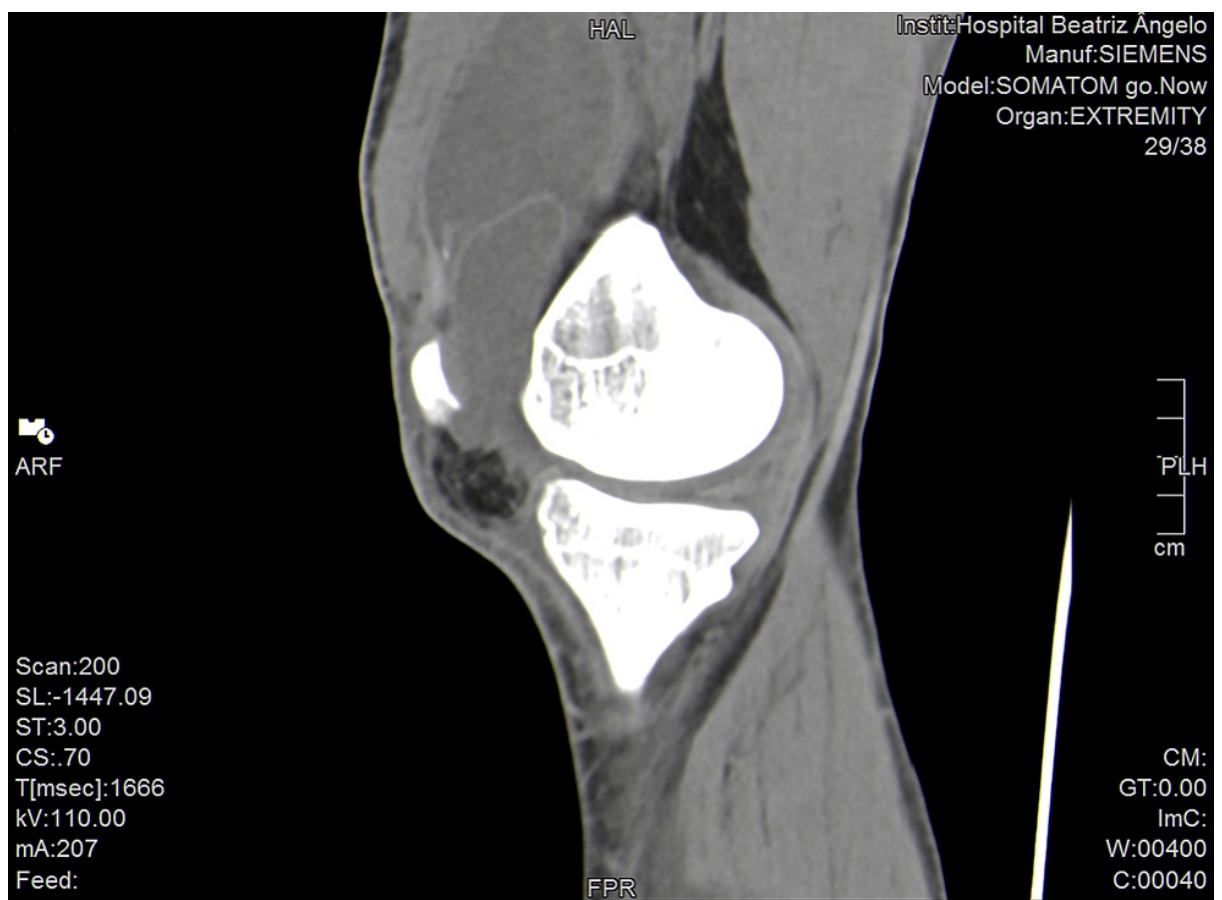

FIGURE 2: Subquadricipital space with septae forming two loci filled with fluid (sagital view). 


\section{Cureus}

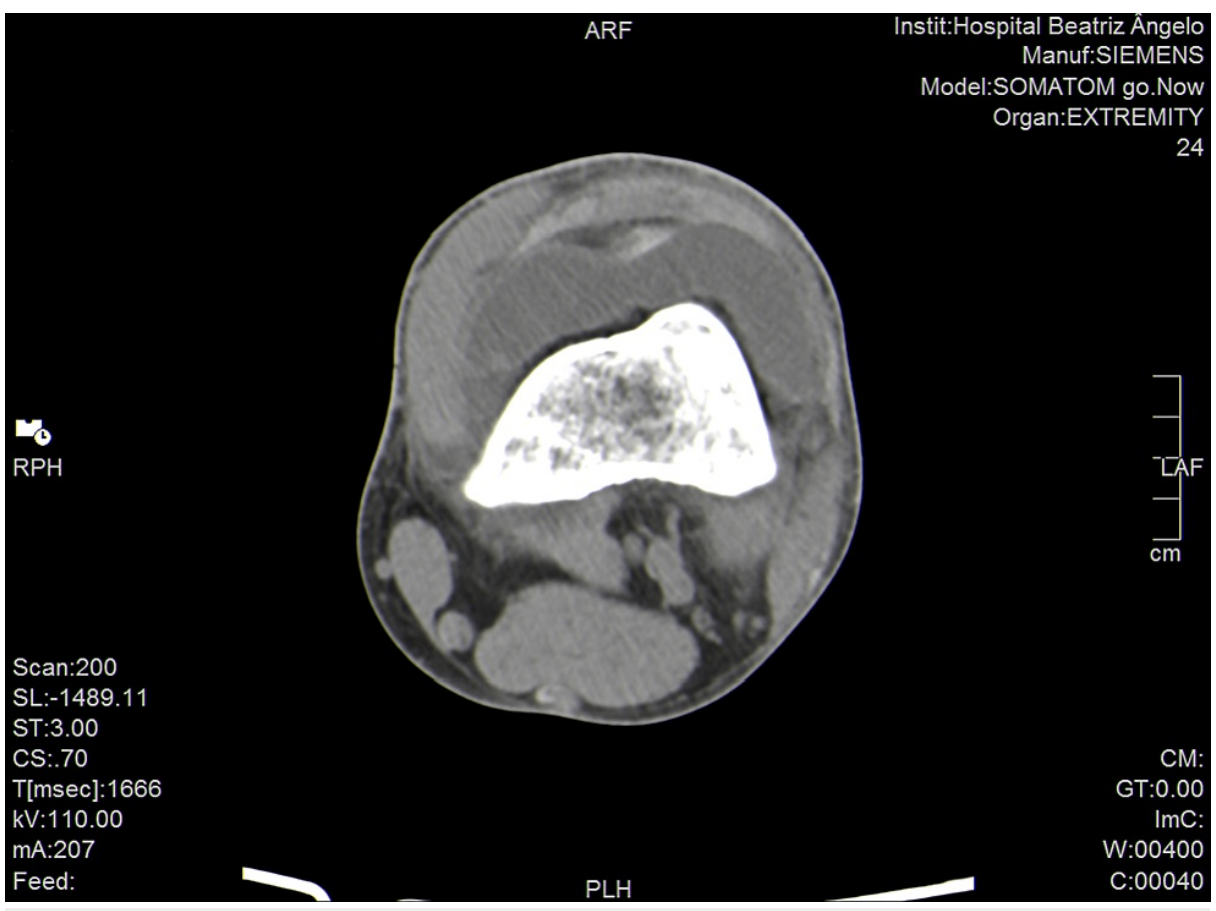

FIGURE 3: Distal portion of femur, showing intra-articular fluid (axis view).

Upon admission, an arthroscopy and lavage were performed, and fluid was sent for culture and sensitivity testing. No thorn was retrieved, hence no identification was possible. Empirical antibiotic therapy was started with flucloxacillin, pending microbiological results. On the third day of admission, $P$. agglomerans was isolated from the synovial fluid, and after consulting with the antibiotic stewardship team antibiotics were changed back to amoxicillin/clavulanate according to sensitivity testing (Table 1 ).

\begin{tabular}{|l|l|}
\hline Antibiotic susceptibilities & P. agglomerans \\
\hline Amoxicillin/slavulanate & $\mathrm{S}$ \\
Ciprofloxacin & $\mathrm{S}$ \\
Gentamicin & $\mathrm{S}$ \\
Cotrimoxazol & $\mathrm{S}$ \\
Ampicillin & $\mathrm{R}$ \\
Piperacillin/tazobactam & $\mathrm{R}$ \\
\hline
\end{tabular}

\section{TABLE 1: Antibiotic susceptibilities.}

The patient showed clinical improvement and inflammatory markers became negative and was discharged after 10 days.

\section{Discussion}

Monoarthritis of the knee is a common diagnosis in the emergency room setting with a broad variety of possible aetiologies [5]. Traumatic aetiologies are the most frequent, nevertheless, infectious diseases should be suspected in some specific cases [5]. Plant thorn-related arthritis is well described but may be overlooked as a differential diagnosis of monoarticular inflammatory disease.

Thorn arthritis/synovitis are classically considered aseptic with three underlying physiopathological mechanisms: foreign body reaction, toxic substance reaction, or immunological reaction to a foreign body [6]. The common link is inflammation with a certain number of cases being infected [4]. Like usually 
described in non-infectious and infectious plant thorn arthritis/synovitis, our patient presented without fever and with a tender joint that yielded a turbid fluid. Classically, retrieved joint fluid is clearly inflammatory predominantly polymorphonuclear and neutrophilic, rarely lymphocytic [4,6]. Similarly, our patient had a fluid with a high leukocyte count and predominance of polymorphonuclear cells.

Diagnosis is frequently delayed for months or even years, particularly when the initial trauma has been forgotten or if that hypothesis has not been considered upfront [6]. Differently from what is reported in the literature, where most cases present in a subacute/chronic fashion [4-6], in the present case, we had a patient with an acute presentation favouring the link between the thorn and the clinical picture. Additionally, arthritis secondary to plant thorn prick is an established medical condition occurring, mostly in children [5] and not adults-like our case. In our case, no thorn was retrieved from arthroscopy, hence a clinical presumptive diagnosis was made since there was a confirmed history of a bramble thorn penetrating injury that the patient remembered accurately and the identification of a thorn-related pathogen like $P$. agglomerans.

The possibility of septic arthritis caused by a slow-growing microorganism is the main concern in the differential diagnosis (Mycobacteria or Enterobacterales). In most cases, the cultural examination is sterile, however, rarely, it grows gram-negative bacteria, more commonly $P$. agglomerans [6]. In our case, microbiological results were considerably faster than expected, which might be attributable to faster microbiological detection methods and/or clinical suspicion since trauma with a thorn was taken into account from the beginning.

$P$. agglomerans antimicrobial sensitivity is variable, it is frequently resistant to $\beta$-lactams [6]. P. agglomerans and other Enterobacterales may produce $\beta$-lactamase and are not always covered by the conventional empirical antibiotic treatment schemes for septic arthritis [1]. In previous studies, $P$. agglomerans has shown sensitivity to amikacin, gentamicin, carbapenems, cotrimoxazole, ampicillin/sulbactam, cephalosporines, and quinolones [6]. However, in our case, we had confirmation of susceptibility to amoxicillin/clavulanate hence the choice to change back from flucloxacillin. In line with the previously described case series [1], antibiotic therapy alone was ineffective in this case, as evidenced by the patient's return to the emergency room with a tense joint after starting antibiotics, necessitating an arthroscopy and lavage.

\section{Conclusions}

This case highlights the importance of precise and thorough medical history, especially for less common presentations. It also shows how source control is a cornerstone of septic arthritis and the importance of microbiology results for adequate antibiotic therapy.

As with other infections, antibiotic therapy should be initiated as soon as possible. Empirical therapy should begin as soon as fluids for culture are harvested and should be adjusted, according to microbiological reports. If source control cannot be achieved and antibiotic treatment fails, medical history should be clarified while keeping a high suspicion for an occult foreign body. Early open debridement of the joint with an extensive search for the thorn seems justified when the culture of synovial fluid yields P. agglomerans.

\section{Additional Information \\ Disclosures}

Human subjects: Consent was obtained or waived by all participants in this study. Conflicts of interest: In compliance with the ICMJE uniform disclosure form, all authors declare the following: Payment/services info: All authors have declared that no financial support was received from any organization for the submitted work. Financial relationships: All authors have declared that they have no financial relationships at present or within the previous three years with any organizations that might have an interest in the submitted work. Other relationships: All authors have declared that there are no other relationships or activities that could appear to have influenced the submitted work.

\section{References}

1. Koester T, Kusano T, Eijer H, Escher R, Waldegg G: Septic arthritis of the knee due to Pantoea agglomerans: look for the thorn. J Bone Jt Infect. 2020, 6:51-5. 10.5194/jbji-6-51-2020

2. De Champs C, Le Seaux S, Dubost JJ, Boisgard S, Sauvezie B, Sirot J: Isolation of Pantoea agglomerans in two cases of septic monoarthritis after plant thorn and wood sliver injuries. J Clin Microbiol. 2000, 38:460-1. 10.1128/JCM.38.1.460-461.2000

3. Dutkiewicz J, Mackiewicz B, Kinga Lemieszek M, Golec M, Milanowski J: Pantoea agglomerans: a mysterious bacterium of evil and good. Part III. Deleterious effects: infections of humans, animals and plants. Ann Agric Environ Med. 2016, 23:197-205. 10.5604/12321966.1203878

4. Duerinckx JF: Case report: subacute synovitis of the knee after a rose thorn injury: unusual clinical picture . Clin Orthop Relat Res. 2008, 466:3138-42. 10.1007/s11999-008-0482-2

5. Ramos JT, Barbosa N, Almeida J, Karmali S, Guerreiro R, Magalhães G: Knee septic arthritis after palm tree thorn injury: a case report. J Orthop Case Rep. 2021, 11:13-5. 10.13107/jocr.2021.v11.i03.2066

6. Fianyo E, Guignard S, Economu A, Thellier N, Davidowicz K, Chevalier X: Thorn synovitis: report of 2 cases. 


\section{Cureus}

Rev Med Interne. 2015, 36:426-9. 10.1016/j.revmed.2014.05.021 